\title{
Transport of interacting electrons in arrays of quantum dots and diffusive wires
}

\author{
Dmitri S. Golubev ${ }^{1,3}$ and Andrei D. Zaikin ${ }^{2,3}$ \\ ${ }^{1}$ Institut für Theoretische Festkörperphysik, Universität Karlsruhe, 76128 Karlsruhe, Germany \\ ${ }^{2}$ Forschungszentrum Karlsruhe, Institut für Nanotechnologie, 76021, Karlsruhe, Germany \\ ${ }^{3}$ I.E. Tamm Department of Theoretical Physics, P.N. Lebedev Physics Institute, 119991 Moscow, Russia
}

\begin{abstract}
We develop a detailed theoretical investigation of the effect of Coulomb interaction on electron transport in arrays of chaotic quantum dots and diffusive metallic wires. Employing the real time path integral technique we formulate a new Langevin-type of approach which exploits a direct relation between shot noise and interaction effects in mesoscopic conductors. With the aid of this approach we establish a general expression for the Fano factor of 1D quantum dot arrays and derive a complete formula for the interaction correction to the current which embraces all perturbative results previously obtained for various quasi-0D and quasi-1D disordered conductors and extends these results to yet unexplored regimes.
\end{abstract}

\section{INTRODUCTION}

Recently a profound relation was elucidated ${ }^{1,2.3 .4}$ between full counting statistics (FCS $)^{\frac{5}{}}$ and electronelectron interaction effects in coherent mesoscopic conductors. In particular, it was demonstrated 1 that the leading interaction correction to the current through such conductors is determined by the second cumulant of the current operator $\mathcal{S}_{2}$, i.e. by the power spectrum of the shot noise ${ }^{6}$. The interaction correction to the shot noise $\mathcal{S}_{2}$ was in turn found ${ }^{2}$ proportional to the third cumulant of the current operator $\mathcal{S}_{3}$. Even more generally, one can show $^{3,4}$ that the lowest order interaction correction to the $n$-th current cumulant $\mathcal{S}_{n}$ is determined by $\mathcal{S}_{n+1}$ for all values of $n$. Since the current cumulants in the absence of interactions can be conveniently analyzed within the FCS formalism ${ }^{5}$, the above observations provide a great deal of information about the effect of electron-electron interactions as well.

In order to investigate the influence of interactions on higher current cumulants it is in general necessary to employ a complete expression for the effective action of a coherent scatterer ${ }^{2.3 .4}$. At the same time the results 1 for the first cumulant, i.e. the relation between the leading interaction correction to the current and the shot noise can be understood already within a simple and transparent theoretical framework of quasiclassical Langevin equations. In the case of a single coherent scatterer shunted by some linear Ohmic resistor $R_{S}$ these equations take a remarkably simple form

$$
\begin{aligned}
C \frac{\ddot{\varphi}}{e}+\frac{1}{R} \frac{\dot{\varphi}}{e} & =I(t)+\xi(t), \\
\frac{1}{R_{S}}\left(V_{x}-\frac{\dot{\varphi}}{e}\right) & =I(t)+\xi_{S}(t) .
\end{aligned}
$$

Here $C$ is the scatterer capacitance, $\dot{\varphi} / e=V$ is the fluctuating voltage across the scatterer and $V_{x}$ is the total voltage applied to the system "scatterer+shunt". As usually, one describes the scatterer by a set of conducting channels with transmissions $T_{k}$. The scatterer conductance is then defined by means of the standard Landauer formula

$$
\frac{1}{R}=\frac{e^{2}}{\pi} \sum_{k} T_{k}
$$

$\xi(t)$ is the noise of the scatterer, characterized by the correlator

$$
\begin{aligned}
\left\langle\xi\left(t_{1}\right) \xi\left(t_{2}\right)\right\rangle= & \frac{1-\beta+\beta \cos \left[\varphi\left(t_{1}\right)-\varphi\left(t_{2}\right)\right]}{R} \\
& \times \int \frac{d \omega}{2 \pi} \omega \operatorname{coth} \frac{\omega}{2 T} \mathrm{e}^{-i \omega\left(t_{1}-t_{2}\right)},
\end{aligned}
$$

where

$$
\beta=\frac{\sum_{k} T_{k}\left(1-T_{k}\right)}{\sum_{k} T_{k}}
$$

is the Fano factor, and $\xi_{S}(t)$ is the equilibrium noise of the shunt with the correlator

$$
\left\langle\xi_{S}\left(t_{1}\right) \xi_{S}\left(t_{2}\right)\right\rangle=\frac{1}{R_{S}} \int \frac{d \omega}{2 \pi} \omega \operatorname{coth} \frac{\omega}{2 T} \mathrm{e}^{-i \omega\left(t_{1}-t_{2}\right)} .
$$

The whole approach based on Eqs. (15) is applicable either at sufficiently high energies or, more importantly, if at least one of the two dimensionless conductances, $g=2 \pi / e^{2} R$ and/or $g_{S}=2 \pi / e^{2} R_{S}$, remains large. The above Langevin equations make the relation between the interaction correction to the current and the shot noise completely transparent demonstrating that the former can be derived just if one accounts for the noise contribution in the equation describing the balance of currents across the scatterer.

The above simple approach may hold only for relatively compact scatterers, in which case the description of interaction effects with the aid of the uniform in space fluctuating field $\varphi$ is sufficient. For spatially extended conductors the coordinate dependence of this field cannot anymore be disregarded and the whole analysis needs to be modified. This modification is trivial if one considers an array of scatterers connected between each other by relatively big metallic grains. Assuming that the electron distribution function in each of these grains remains in 
equilibrium one can describe the $n$-th scatterer by its own fluctuating field $\varphi_{n}$ which obeys the same set of Langevin equations (11), (3). For arrays of tunnel junctions this approach was employed in Ref. 7. The corresponding generalization of the results ${ }^{7}$ to the case of arbitrary scatterers just requires modification of the Fano factor in the noise correlator (3).

The condition of local equilibrium inside metallic grains implies that the corresponding electron dwell time $\tau_{D}$ between two adjacent scatterers should be much longer than the inelastic relaxation time $\tau_{\text {in }}$. If this condition is violated, the electron distribution function is driven out of equilibrium and the whole consideration becomes more complicated. In the case of a quantum dot formed by two arbitrary scatterers the latter situation was analyzed in details in Ref. 8 and also in Refs. 49 for the case of chaotic dots. In all these works it was demonstrated that in the limit of large conductances and in the voltage biased regime the interaction correction to the conductance tends to saturate at temperatures/voltages below $1 / \tau_{D}$. This implies that for finite values of $\tau_{D}$ highly conducting quantum dots should show metallic behavior down to zero temperature.

It is important to emphasize that this observation holds only provided the voltage source is attached $d i$ rectly to the quantum dot, i.e. the external impedance is equal to zero. For non-zero external impedances voltage fluctuations lift the conductance saturation, and the amplitude of the interaction correction keeps increasing with decreasing $T$ even at temperatures well below $1 / \tau_{D}$. In this regime the interaction correction was found $\underline{8}$ to scale linearly with the total Fano factor of the quantum dot and to depend logarithmically on temperature/voltage for sufficiently large external impedances or if this impedance is purely Ohmic.

For similar reasons no saturation of the interaction correction at energies below $1 / \tau_{D}$ should be expected for chains and arrays of quantum dots. Recently this situation was analyzed diagrammatically 10 in the case of granular tunnel junction arrays. Indeed, it was found that the interaction correction increases with decreasing temperature both above and below the inverse dwell time in individual grains. At $T<1 / \tau_{D}$ the authors 10 recovered exactly the same expression for the interaction correction as that known in the case of diffusive conductors 11 . This equivalence is by no means surprising if one bears in mind the fundamental relation between the interaction correction and the shot noise on one hand, and the results 12,13 on the other hand, which demonstrate that the shot noise of a sufficiently long array of tunnel junctions is equivalent to that of a diffusive wire. Extending these arguments to arbitrary scatterers, with the aid of the results 13 one can anticipate that at sufficiently low energies $\left(\lesssim 1 / \tau_{D}\right)$ and large spatial scales the interaction correction should be described universally for any array of quantum dots and ultimately for any mesoscopic conductor in the metallic regime. This universality will indeed be demonstrated below.
The main goal of the present paper is to generalize the simple Langevin equation approach ${ }^{7}$ to situations in which relaxation of the electron distribution function occurs at much longer time scales as compared to the electron dwell time between two adjacent scatterers $\tau_{D} \ll \tau_{\text {in }}$. Although the distribution function may significantly deviate from the Fermi function, it is possible to account for these deviations within the (generalized) Langevin equation analysis and to formulate a closed set of equations which fully determine the interaction correction to the $I-V$ curve of disordered conductors.

The structure of the paper is as follows. In Sec. II we will specify the model of a disordered metallic conductor and present a phenomenological derivation of the basic Langevin equations for our problem. This derivation will be carried out with the aid of simple and transparent physical arguments which make the whole approach easy to understand without going into technical details. A more advanced analysis employing the effective action technique will be described in Sec. III. This analysis provides rigorous justification for our phenomenological derivation and allows to illustrate a useful relation between our technique and the classical BoltzmannLangevin approach ${ }^{6}$. In Sec. IV we will probe our Langevin technique by explicitly deriving the shot noise spectrum and the Fano factor for arrays of chaotic quantum dots in the absence of interactions. The remainder of the paper will be devoted to the analysis of the leading interaction correction to the current in arrays of quantum dots and mesoscopic diffusive wires. In Sec. $\mathrm{V}$ we will derive the general expression for this correction which then will be applied to homogeneous arrays of quantum dots in Sec. VI. In the latter case we will establish a complete analytic form of the interaction correction and present the corresponding simplified expressions in a number of important limits. Our general formula, Eq. (63), embraces all previous results 1.4.7.8.9.10.11.14 obtained in various types of quasi-0D and quasi-1D disordered conductors, allows to establish a transparent relation between these results and to extend them to yet unexplored regimes. A brief analysis of an additional effect of external leads will be presented in Sec. VII. We will then discuss our results and conclude the paper in Sec. VIII.

\section{THE MODEL AND PHENOMENOLOGICAL ANALYSIS}

We shall consider a chain of $N-1$ quantum dots as it is shown in Fig. 1. Each dot can be viewed as an island in-between two scatterers/barriers which in turn connect adjacent quantum dots. Electrons can enter the dot through one of the barriers, spend some time there propagating between the barriers, possibly being scattered at the barriers, outer walls or otherwise, and finally leave the dot through another barrier. In what follows we will adopt the model of chaotic quantum dots. 


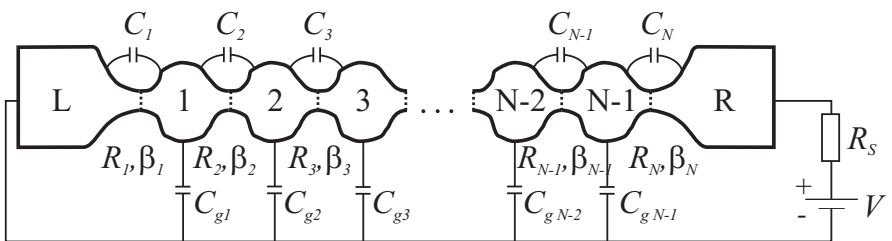

FIG. 1: 1D array of chaotic quantum dots. The array consists of $N-1$ dots and $N$ barriers. The $n$-th dot is characterized by mean level spacing $\delta_{n}$ and gate capacitance $C_{g n}$. The $n$ th barrier is described by its Landauer conductance $1 / R_{n}$, capacitance $C_{n}$ and Fano factor $\beta_{n}$. The array is placed inbetween two big metallic reservoirs which are connected to the voltage source via Ohmic resistor $R_{S}$.

Each of the $N$ barriers will be described by its Landauer conductance $1 / R_{n}=\left(e^{2} / \pi\right) \sum_{k} T_{k}^{(n)}$, capacitance $C_{n}$ and Fano factor $\beta_{n}=\sum_{k} T_{k}^{(n)}\left(1-T_{k}^{(n)}\right) / \sum_{k} T_{k}^{(n)}$, where $T_{k}^{(n)}$ is the transmission of the $k$-th conducting mode in the $n$-th barrier. We also define dimensionless conductances of the scatterers $g_{n}=2 \pi / e^{2} R_{n}$. In what follows we will assume that each scatterer has many conducting channels and that its dimensionless conductance is large $g_{n} \gg 1$. The $n$-th dot will be characterized by the mean level spacing $\delta_{n}=1 / N_{0} \mathcal{V}_{n}$, where $\mathcal{V}_{n}$ is the dot volume and $N_{0}$ is the density of states at the Fermi level. For the sake of generality we will also assume that each dot has an additional capacitance to the ground $C_{g n}$. Finally, the first and the last scatterers are connected to two big metallic reservoirs which in turn are connected to the voltage source via external leads with an Ohmic resistance $R_{S}$.

An important assumption concerns the spatial dependence of fluctuating voltages in our system. Similarly to Ref. 8 we will allow for voltage drops $V_{n}(t)$ only across the barriers, while inside the dots voltages can depend arbitrarily on time but not on the spatial coordinates. This assumption is usually well satisfied for metallic dots considered here. In the leads the voltage fields are assumed to vary slowly in space. In the course of our derivation we will essentially neglect all mechanisms of inelastic relaxation which are not related to electron-electron interactions. We will also disregard weak localization effects which can be easily suppressed, e.g., by externally applied magnetic field.

We will now proceed with our phenomenological analysis of the above model.

\section{A. Noise correlator}

As a first step we will specify the general expression for the noise correlator needed for our derivation. Let us assume that the electron distribution function $f_{n}(E)$ in the $n$-th dot does not depend on time for all $n$ but may deviate arbitrarily from the Fermi function. Below we also assume that the electron energies are measured with respect to the unique reference energy for the whole array. In this case the noise of the $n$-th scatterer $\mathcal{S}_{n}\left(t_{1}, t_{2}\right)=$ $\left\langle\xi_{n}\left(t_{1}\right) \xi_{n}\left(t_{2}\right)\right\rangle$ takes the form ${ }^{6}$

$$
\begin{array}{r}
\mathcal{S}_{n}\left(t_{1}, t_{2}\right)=\frac{1}{2 R_{n}} \int \frac{d \omega}{2 \pi} \int d E \mathrm{e}^{-i \omega\left(t_{1}-t_{2}\right)} \\
\times\left\{\beta _ { n } \left[f_{n-1}(E) h_{n}(E+\omega)+f_{n}(E+\omega) h_{n-1}(E)\right.\right. \\
\left.+f_{n}(E) h_{n-1}(E+\omega)+f_{n-1}(E+\omega) h_{n}(E)\right] \\
+\left(1-\beta_{n}\right)\left[f_{n-1}(E) h_{n-1}(E+\omega)\right. \\
+f_{n-1}(E+\omega) h_{n-1}(E)+f_{n}(E) h_{n}(E+\omega) \\
\left.\left.+f_{n}(E+\omega) h_{n}(E)\right]\right\},
\end{array}
$$

where $h_{n}(E)=1-f_{n}(E)$. Let us define the function

$$
G_{n}\left(t_{1}, t_{2}\right)=\int \frac{d E}{2 \pi} \mathrm{e}^{-i E\left(t_{1}-t_{2}\right)}\left(1-2 f_{n}(E)\right) .
$$

In stationary situations this function depends only on the time difference $t_{1}-t_{2}$ and it also obeys the condition $G_{n}\left(t_{2}, t_{1}\right)=G_{n}^{*}\left(t_{1}, t_{2}\right)$. In equilibrium the distribution function equals to the Fermi function

$$
f_{F}(E)=\frac{1}{1+\exp \left(\frac{E}{T}\right)} .
$$

Substituting this function into Eq. (77) one finds $G_{n}\left(t_{1}, t_{2}\right)=-i T \operatorname{Re}\left[1 / \sinh \pi T\left(t_{1}-t_{2}+i \epsilon\right)\right]$, where $\epsilon$ is an infinitesimal positive constant. At $t_{1} \rightarrow t_{2}$ one gets $G_{n}\left(t_{1}, t_{2}\right) \rightarrow-i \operatorname{Re}\left[1 / \pi\left(t_{1}-t_{2}+i \epsilon\right)\right]$. This analytical property turns out to be general, i.e. it equally applies both to equilibrium and non-equilibrium situations. Expressing Eq. (6) via $G_{n}\left(t_{1}, t_{2}\right)$, we obtain

$$
\begin{aligned}
\mathcal{S}_{n}\left(t_{1}, t_{2}\right) & =\frac{1}{\pi R_{n}} \frac{\epsilon^{2}}{\left(\left(t_{1}-t_{2}\right)^{2}+\epsilon^{2}\right)^{2}} \\
-\frac{\pi \beta_{n}}{2 R_{n}}\left[G_{n-1}\left(t_{1}, t_{2}\right) G_{n}\left(t_{2}, t_{1}\right)+G_{n}\left(t_{1}, t_{2}\right) G_{n-1}\left(t_{2}, t_{1}\right)\right] & \\
- & \frac{\pi\left(1-\beta_{n}\right)}{2 R_{n}}\left[\left|G_{n-1}\left(t_{1}, t_{2}\right)\right|^{2}+\left|G_{n}\left(t_{1}, t_{2}\right)\right|^{2}\right] .
\end{aligned}
$$

Although the formula (9) has been derived under the assumption that the distribution function does not depend on time, we will show later that it remains valid also in non-stationary situations. In the latter case the function $G_{n}\left(t_{1}, t_{2}\right)$ can be understood as the Keldysh component of the quasiclassical Usadel Green function.

\section{B. Kinetic equation}

Our next step is to derive the kinetic equation for the function $G_{n}\left(t_{1}, t_{2}\right)$. For simplicity we again start from the stationary situation, in which case fluctuations of voltages in our system can be neglected. In what follows we will assume that both $f_{n}(E)$ and $G_{n}\left(t_{1}, t_{2}\right)$ do not depend on coordinates inside the $n$-th dot. The total number of 
electrons with energies in the interval $[E, E+d E]$ in the $n$-th dot is $2 N_{0} \mathcal{V}_{n} f_{n}(E) d E$, where the factor 2 accounts for spin degeneracy. This number may change in time only if electrons leave and/or enter the dot trough the left $(n-$ th $)$ and the right $(n+1-$ th) barriers. One finds

$$
\frac{2 d E}{\delta_{n}} \frac{\partial f_{n}(t, E)}{\partial t}=J_{n}^{\text {in }}-J_{n}^{\text {out }} .
$$

The escape rate from the $n$-th dot and the transition rate to this dot - both through the $n$-th scatterer - are respectively

$$
\Gamma_{n-1, n}=g_{n} \delta_{n} / 4 \pi, \quad \Gamma_{n, n-1}=g_{n} \delta_{n-1} / 4 \pi .
$$

Then for $J_{n}^{\text {out }}$ one obtains

$$
J_{n}^{\text {out }}=2\left(\Gamma_{n+1, n}+\Gamma_{n-1, n}\right) f_{n}(E) d E / \delta_{n},
$$

and similarly for $J_{n}^{i n}$. Combining the above simple expressions we arrive at the kinetic equation

$$
\frac{4 \pi}{\delta_{n}} \frac{\partial f_{n}}{\partial t}=-\left(g_{n}+g_{n+1}\right) f_{n}+g_{n} f_{n-1}+g_{n+1} f_{n+1},
$$

where $1 \leq n \leq N-1$. The boundary conditions to this kinetic equation are set by the requirement that the distribution functions in the left and the right reservoirs, $f_{0}$ and $f_{N}$, are equal to the Fermi function, i.e.

$$
f_{0}(E)=f_{F}(E), \quad f_{N}(E)=f_{F}(E-e V) .
$$

Here and below $V$ is the total voltage applied to the array. We note that the function $1-2 f_{n}$ also satisfies Eq. (12).

The kinetic equation for the function $G_{n}\left(t_{1}, t_{2}\right)$ can be obtained from Eq. (12) if we identify $t=\left(t_{1}+t_{2}\right) / 2$, introduce $s=t_{1}-t_{2}$ and make the Fourier transform of (12) by taking the integral $\int \frac{d E}{2 \pi} \mathrm{e}^{-i E s}(\ldots)$. Then we obtain

$$
\begin{aligned}
\frac{4 \pi}{\delta_{n}} \frac{\partial G_{n}(t, s)}{\partial t}= & -\left(g_{n}+g_{n+1}\right) G_{n}(t, s)+g_{n} G_{n-1}(t, s) \\
& +g_{n+1} G_{n+1}(t, s) .
\end{aligned}
$$

As we have already pointed out Eq. (14) applies only in stationary situations. A proper generalization of this equation for non-stationary cases can be achieved with the aid of general gauge invariance arguments which yield

$$
\begin{array}{r}
\frac{4 \pi}{\delta_{n}}\left(\frac{\partial}{\partial t_{1}}+\frac{\partial}{\partial t_{2}}+i \dot{\Phi}_{n}\left(t_{1}\right)-i \dot{\Phi}_{n}\left(t_{2}\right)\right) G_{n}\left(t_{1}, t_{2}\right) \\
=-\left(g_{n}+g_{n+1}\right) G_{n}\left(t_{1}, t_{2}\right)+g_{n} G_{n-1}\left(t_{1}, t_{2}\right) \\
+g_{n+1} G_{n+1}\left(t_{1}, t_{2}\right)
\end{array}
$$

where we defined $\dot{\Phi}_{n}(t)=\sum_{j=1}^{n} e V_{j}(t)$. This kinetic equation holds for arbitrary time dependent voltages. As before, the boundary conditions to this equation read

$$
\begin{aligned}
G_{0}\left(t_{1}, t_{2}\right) & =-i T \operatorname{Re} \frac{1}{\sinh \pi T\left(t_{1}-t_{2}+i \epsilon\right)}, \\
G_{N}\left(t_{1}, t_{2}\right) & =-i T \mathrm{e}^{-i e V\left(t_{1}-t_{2}\right)} \operatorname{Re} \frac{1}{\sinh \pi T\left(t_{1}-t_{2}+i \epsilon\right)}(.16)
\end{aligned}
$$

\section{Balancing fluctuating charges and voltages}

In order to complete our simple analysis we formulate the standard circuit theory equations, which allow to include the effect of charge accumulation in quantum dots. Let us define the fluctuating excess charge in the $n$-th dot $q_{n}$. We assume that all quantum dots are well described by the capacitance model, in which case one finds

$$
q_{n}=C_{n+1} V_{n+1}-C_{n} V_{n}-C_{g n} \sum_{j=1}^{n} V_{j} .
$$

Here $C_{n}$ is the capacitance of the $n$-th barrier and $C_{g n}$ is the capacitance of the $n$-th dot to the ground. The current $I_{n}$ is formed by the sum of three different terms, namely the standard Ohmic term $V_{n} / R_{n}$, the "kinetic" term $\Gamma_{n, n-1} q_{n-1}-\Gamma_{n-1, n} q_{n}$ and the noise term $\xi_{n}$ :

$$
I_{n}=\frac{V_{n}}{R_{n}}+\Gamma_{n, n-1} q_{n-1}-\Gamma_{n-1, n} q_{n}+\xi_{n} .
$$

The variation of the charge $q_{n}$ is in turn determined by the currents flowing through the $n$-th and $n+1-$ th barriers. We get

$$
\dot{q}_{n}=I_{n}-I_{n+1} .
$$

Finally, the sum of all fluctuating voltages $V_{n}$ should be equal to the total applied voltage,

$$
\sum_{n=1}^{N} V_{n}=V
$$

Eqs. 9151720 form a complete set of equations, which allow to find the first order interaction correction to the $I-V$ characteristics for an array of quantum dots. These equations represent a straightforward generalization of the Langevin approach employed in Ref. 7. In contrast to the latter, however, our present analysis accounts for the electron dwell time in quantum dots and also non-perturbatively treats electron transport through the scatterers. In the limit of long dwell times $\Gamma_{n \pm 1, n} \ll 1 / \tau_{\text {in }}$ and small channel transmissions $\beta_{n} \rightarrow 1$ (i.e. for tunnel junction arrays) our equations are replaced by those of Ref. 7 .

\section{Interaction correction and shot noise}

Finally, let us establish an important relation between interaction correction to the current and the shot noise. Performing summation of Eqs. (18) with the weights $R_{n}$, we obtain

$$
I=\frac{V}{R_{\Sigma}}+\sum_{n=1}^{N} \frac{R_{n}\left\langle\xi_{n}\right\rangle}{R_{\Sigma}}
$$


This formula generalizes our previous results derived for a coherent scatterer $\frac{1}{\underline{1}}$ and a quantum $\operatorname{dot}^{\underline{8}}$ to the case of quantum dot arrays and spatially extended disordered conductors. Eq. (21) demonstrates that the interaction correction to the $I-V$ curve of an array of scatterers scales linearly with the current noise produced by these scatterers. In the absence of noise the interaction correction is identically zero, and the standard Ohm's law is recovered. Eq. 211 will be extensively used in our subsequent calculation.

\section{RIGOROUS DERIVATION}

The phenomenological analysis presented in the previous section clearly illustrates the relation between shot noise and interaction effects in electron transport. Now we will demonstrate that Eqs. (91519) can also be derived within the framework of a rigorous quantum mechanical procedure. This derivation will also allow to determine the validity range of our Langevin approach.

We first note that for a particular case of two scatterers the above equations follow from the effective action analysis ${ }^{8}$ after averaging of the action over mesoscopic fluctuations. Below we will see that for the case $N=2$ these equations yield exactly the same results as those derived in Ref. 8 for chaotic quantum dots. Direct generalization of the method ${ }^{8}$ to the case of quantum dot arrays, though technically possible, turns out to be rather involved since one should first establish the full quantum mechanical action for the whole array and then perform its averaging over mesoscopic fluctuations. In this case it appears more convenient to average the action already at the first stage of the calculation. In order to accomplish this goal we will employ the non-linear $\sigma$-model-type of approach combined with the Keldysh technique. This method was proposed in Ref. 15 and recently applied to chaotic quantum dots in Ref. 4. Below we will extend this technique to arrays of quantum dots.

\section{A. Effective action}

In the presence of electron-electron interactions general quantum mechanical description of both compact scatterers 1.2 .3 .4 .16 and extended disordered conductors $\frac{15.17}{}$ can be formulated in terms of the effective action which depends on the fluctuating HubbardStratonovich fields $V_{1}$ and $V_{2}$ defined on the two branches of the Keldysh contour. In the situation considered here the action also depends on the fluctuating Green function $\check{Q}_{n}$ which is $2 \times 2$ matrix in Keldysh space satisfying the normalization condition

$$
\check{Q}^{2}\left(t_{1}, t_{2}\right)=\int d t_{3} \check{Q}\left(t_{1}, t_{3}\right) \check{Q}\left(t_{3}, t_{2}\right)=\delta\left(t_{1}-t_{2}\right) \check{1},
$$

and on the fluctuating phases of the dots

$$
\check{\Phi}_{n}=\Phi_{n} \check{1}+\frac{\Phi^{-}}{2} \check{\sigma}_{z},
$$

where we defined

$$
\begin{array}{r}
\Phi_{n}=\sum_{j=1}^{n} \int_{t_{0}}^{t} d t^{\prime} e\left(V_{j, 1}\left(t^{\prime}\right)+V_{j, 2}\left(t^{\prime}\right)\right) / 2, \\
\Phi_{n}^{-}=\sum_{j=1}^{n} \int_{t_{0}}^{t} d t^{\prime} e\left(V_{j, 1}\left(t^{\prime}\right)-V_{j, 2}\left(t^{\prime}\right)\right) .
\end{array}
$$

Here and below $\check{\sigma}_{x, y, z}$ are the Pauli matrices in Keldysh space.

The complete expression for the effective action of the array reads

$$
\begin{aligned}
i S= & i \sum_{n=1}^{N} \int_{0}^{t} d t^{\prime} \frac{C_{n} \dot{\varphi}_{n} \dot{\varphi}_{n}^{-}}{e^{2}} \\
& +i \sum_{n=1}^{N-1} \int_{0}^{t} d t^{\prime}\left(\frac{C_{g n}}{e^{2}}+\frac{2}{\delta_{n}}\right) \dot{\Phi}_{n} \dot{\Phi}_{n}^{-} \\
& +\sum_{n=1}^{N} \frac{1}{2} \operatorname{Tr} \ln \left[1+\frac{T_{k}^{(n)}}{4}\left(\left\{\check{Q}_{n-1}, \check{Q}_{n}\right\}-2\right)\right] \\
& -\sum_{n=1}^{N-1} \frac{2 \pi i}{\delta_{n}} \operatorname{Tr}\left(\left(i \frac{\partial}{\partial t}-\dot{\Phi}_{n}-\frac{\dot{\Phi}_{n}^{-}}{2} \check{\sigma}_{z}\right) \check{Q}_{n}\right)
\end{aligned}
$$

Here the trace includes the summation over the channel index $k$ while the superscript $n$ indicates the scatterer number. The boundary conditions for the operators $\check{Q}$ $\operatorname{are}^{\underline{4}}$

$$
\begin{aligned}
\check{Q}_{0}\left(t_{1}, t_{2}\right)= & \frac{-i T\left(\check{\sigma}_{z}+i \check{\sigma}_{y}\right)}{\sinh \pi T\left(t_{1}-t_{2}\right)}-\delta\left(t_{1}-t_{2}\right) \check{\sigma}_{x}, \\
\check{Q}_{N}\left(t_{1}, t_{2}\right)= & \frac{-i T \mathrm{e}^{-i e V\left(t_{1}-t_{2}\right)}\left(\check{\sigma}_{z}+i \check{\sigma}_{y}\right)}{\sinh \pi T\left(t_{1}-t_{2}\right)} \\
& -\delta\left(t_{1}-t_{2}\right) \check{\sigma}_{x} .
\end{aligned}
$$

Physical observables can be evaluated by means of the following equation:

$$
\langle\hat{A}\rangle=\int_{\check{Q}_{n}^{2}=\check{1}} \mathcal{D} \check{Q}_{n} \int \mathcal{D} \Phi \mathcal{D} \Phi^{-} A\left(\frac{\delta}{\delta \Phi}, \frac{\delta}{\delta \Phi^{-}}\right) \mathrm{e}^{i S} .
$$

In the FCS-type of approach ${ }^{3.4}$ the matrix $\check{Q}_{n}$ has the form

$$
\check{Q}_{n}=\mathrm{e}^{i \chi_{n} \check{\sigma}_{z} / 2}\left[\hat{G}_{n}\left(\check{\sigma}_{z}+i \check{\sigma}_{y}\right)-\check{\sigma}_{x}\right] \mathrm{e}^{-i \chi_{n} \check{\sigma}_{z} / 2},
$$

where $\chi_{n}$ is the time and space independent "counting field" for the $n$-th quantum dot. In our problem $\chi_{n}$ has to be replaced by an arbitrary fluctuating Hermitian operator. This observation suggests the following parametrization of the operator $\check{Q}_{n}$ :

$$
\check{Q}_{n}=\mathrm{e}^{i \hat{W}_{n} \check{\sigma}_{z}}\left[\hat{G}_{n}\left(\check{\sigma}_{z}+i \check{\sigma}_{y}\right)-\check{\sigma}_{x}\right] \mathrm{e}^{-i \hat{W}_{n} \check{\sigma}_{z}},
$$


where $\hat{G}_{n}$ and $\hat{W}_{n}$ are Hermitian operators. Here $\hat{G}_{n}$ accounts for fluctuations of the electron distribution function in the $n$-th quantum dot or, more generally, for fluctuations of the Keldysh-Usadel Green function. The operator $\hat{W}_{n}$ describes "quantum" fluctuations of the field $\check{Q}_{n}$. It is possible to demonstrate that an arbitrary operator $\check{Q}_{n}$ satisfying the normalization condition (22) and being sufficiently close to the "classical" one, $\hat{G}_{n}^{(0)}\left(\check{\sigma}_{z}+i \check{\sigma}_{y}\right)-\check{\sigma}_{x}$, can be written in the form (30). We further note that the parametrization (30) is not identical to that proposed in Ref. 15.

Let us expand the action (26) to the second order in the small operators $\hat{W}_{n}$. Then we obtain

$$
\begin{aligned}
i S= & i \sum_{n=1}^{N} \int_{0}^{t} d t^{\prime} \frac{C_{n} \dot{\varphi}_{n} \dot{\varphi}_{n}^{-}}{e^{2}} \\
& +i \sum_{n=1}^{N-1} \int_{0}^{t} d t^{\prime}\left(\frac{C_{g n}}{e^{2}}+\frac{2}{\delta_{n}}\right) \dot{\Phi}_{n} \dot{\Phi}_{n}^{-} \\
& +\sum_{n=1}^{N-1} \frac{2 \pi}{\delta_{n}} \operatorname{Tr}\left(i \dot{\Phi}_{n}^{-} \hat{G}_{n}-2\left[i \frac{\partial}{\partial t}-\dot{\Phi}_{n}, \hat{G}_{n}\right] \hat{W}_{n}\right) \\
& +\sum_{n=1}^{N} g_{n} \operatorname{Tr}\left(-i\left(\hat{G}_{n}-\hat{G}_{n-1}\right) \hat{w}_{n}+\beta_{n} \hat{G}_{n-1} \hat{w}_{n} \hat{G}_{n} \hat{w}_{n}\right. \\
& \left.+\frac{1-\beta_{n}}{2}\left[\left(\hat{G}_{n-1} \hat{w}_{n}\right)^{2}+\left(\hat{G}_{n} \hat{w}_{n}\right)^{2}\right]-\hat{w}_{n}^{2}\right) .
\end{aligned}
$$

where $\hat{w}_{n}=\hat{W}_{n}-\hat{W}_{n-1}$. The quadratic in $\hat{w}_{n}$ terms can be decoupled with the aid of the Hubbard-Stratonovich transformation. One finds

$$
\begin{array}{r}
\exp \left\{\sum _ { n = 1 } ^ { N } g _ { n } \operatorname { T r } \left(\beta_{n} \hat{G}_{n-1} \hat{w}_{n} \hat{G}_{n} \hat{w}_{n}\right.\right. \\
\left.\left.+\frac{1-\beta_{n}}{2}\left[\left(\hat{G}_{n-1} \hat{w}_{n}\right)^{2}+\left(\hat{G}_{n} \hat{w}_{n}\right)^{2}\right]-\hat{w}_{n}^{2}\right)\right\}= \\
\left\langle\mathrm{e}^{-i \sum_{n=1}^{N} \operatorname{Tr}\left(\hat{\zeta}_{n} \hat{w}_{n}\right)}\right\rangle_{\hat{\zeta}_{n}},
\end{array}
$$

where we introduced the Gaussian stochastic operator $\hat{\zeta}_{n}$ with the pair correlator

$$
\begin{array}{r}
\left\langle\zeta_{n}\left(t_{1}, t_{2}\right) \zeta_{n}\left(t_{3}, t_{4}\right)\right\rangle=2 g_{n} \delta\left(t_{3}-t_{2}\right) \delta\left(t_{1}-t_{4}\right) \\
-g_{n} \beta_{n}\left[G_{n-1}\left(t_{3}, t_{2}\right) G_{n}\left(t_{1}, t_{4}\right)\right. \\
\left.+G_{n}\left(t_{3}, t_{2}\right) G_{n-1}\left(t_{1}, t_{4}\right)\right] \\
-g_{n}\left(1-\beta_{n}\right)\left[G_{n}\left(t_{3}, t_{2}\right) G_{n}\left(t_{1}, t_{4}\right)\right. \\
\left.+G_{n-1}\left(t_{3}, t_{2}\right) G_{n-1}\left(t_{1}, t_{4}\right)\right] .
\end{array}
$$

\section{B. Kinetic equation and Boltzmann-Langevin approach}

We are now in a position to derive the equation of motion for the matrix $\hat{G}_{n}$. In the metallic limit $g_{n} \gg 1$ it is sufficient to restrict our analysis to the least action condition $\delta S / \delta \hat{W}_{n}=0$ which yields

$$
\begin{array}{r}
\frac{4 \pi}{\delta_{n}}\left[\frac{\partial}{\partial t}+i \dot{\Phi}_{n}(t), \hat{G}_{n}\right]=g_{n} \hat{G}_{n-1}+g_{n+1} \hat{G}_{n+1} \\
-\left(g_{n}+g_{n+1}\right) \hat{G}_{n}+\hat{\zeta}_{n}-\hat{\zeta}_{n+1} .
\end{array}
$$

Dropping the operator notations one can rewrite the same equation in the form

$$
\begin{array}{r}
\frac{4 \pi}{\delta_{n}}\left(\frac{\partial}{\partial t_{1}}+\frac{\partial}{\partial t_{2}}+i \dot{\Phi}_{n}\left(t_{1}\right)-i \dot{\Phi}_{n}\left(t_{2}\right)\right) G_{n}=g_{n} G_{n-1} \\
+g_{n+1} G_{n+1}-\left(g_{n}+g_{n+1}\right) G_{n}+\zeta_{n}-\zeta_{n+1} .
\end{array}
$$

Under the condition $g_{n} \gg 1$ one can also neglect the noise terms $\zeta_{n}$ in Eq. (34) which can only contribute in higher orders in $1 / g_{n}$. Dropping these terms one observes the equivalence of the equations (34) and (15).

It is also useful to illustrate a simple relation between our Eq. (34) and the standard Boltzmann-Langevin approach ${ }^{6}$, which is frequently used, e.g., for the analysis of the shot noise in disordered conductors. Let us again define $t=\left(t_{1}+t_{2}\right) / 2$ and $s=t_{1}-t_{2}$. We will assume that $G_{n}$ varies slowly with $t$ and $\dot{\Phi}_{n}$ is a slow function of time. Replacing $i \dot{\Phi}_{n}\left(t_{1}\right)-i \dot{\Phi}_{n}\left(t_{2}\right) \rightarrow i \ddot{\Phi}_{n}(t) s$ and performing the Fourier transformation of Eq. (34) with respect to $s$, one arrives at the equation for the distribution function

$$
\begin{array}{r}
\frac{4 \pi}{\delta_{n}}\left(\frac{\partial f_{n}}{\partial t}+\ddot{\Phi}_{n} \frac{\partial f_{n}}{\partial E}\right)=g_{n} f_{n-1}+g_{n+1} f_{n+1} \\
-\left(g_{n}+g_{n+1}\right) f_{n}-\eta_{n}+\eta_{n+1}
\end{array}
$$

where $2 \eta_{n}=\int d s \mathrm{e}^{i E s} \zeta_{n}(t+s / 2, t-s / 2)$ and

$$
\begin{array}{r}
\left\langle\eta_{n}(t, E) \eta_{m}\left(t^{\prime}, E^{\prime}\right)\right\rangle=2 \pi g_{n} \delta_{m n} \delta\left(t-t^{\prime}\right) \delta\left(E-E^{\prime}\right) \\
\times\left\{\beta_{n}\left[f_{n}(t, E) h_{n-1}(t, E)+f_{n-1}(t, E) h_{n}(t, E)\right]\right. \\
\left.\left(1-\beta_{n}\right)\left[f_{n}(t, E) h_{n}(t, E)+f_{n-1}(t, E) h_{n-1}(t, E)\right]\right\} .
\end{array}
$$

Eqs. (35), (36) represent an extension of the BoltzmannLangevin approach ${ }^{6}$ to arrays of quantum dots. Within the framework of the adiabatic approximation the latter approach follows directly from our rigorous analysis.

\section{Excess charges}

Let us now integrate out the field $\Phi_{n}^{-}$. Being performed with the action (26) this integral gives the functional delta-function equivalent to the equation $\delta S / \delta \Phi_{n}^{-}=0$. This equation yields

$$
\begin{array}{r}
-\left(C_{g n}+\frac{2 e^{2}}{\delta_{n}}\right) \sum_{j=1}^{n} \dot{V}_{j}-C_{n} \dot{V}_{n}+C_{n+1} \dot{V}_{n+1} \\
-\frac{2 \pi e}{\delta_{n}} \frac{d}{d t} G_{n}(t, t)=0 .
\end{array}
$$


The last term in Eq. (37) can be expressed via the excess charge $q_{n}$ in the $n$-th dot. In the stationary case this charge variable is defined as follows

$$
\begin{aligned}
q_{n} & =-\frac{2 e}{\delta_{n}} \int d E\left(f_{n}\left(E+\dot{\Phi}_{n}\right)-f_{F}(E)\right) \\
& =\frac{2 \pi e}{\delta_{n}} \lim _{s \rightarrow 0}\left(G_{n}\left(t+\frac{s}{2}, t-\frac{s}{2}\right)+\frac{i}{\pi s}\right)+\frac{2 e \dot{\Phi}_{n}}{\delta_{n}}
\end{aligned}
$$

This definition can be applied to non-stationary situations as well. Then Eq. (37) is replaced by Eq. (17).

Finally, let us consider the limit $t_{1} \rightarrow t_{2}$ in Eq. (34). Setting $t=\left(t_{1}+t_{2}\right) / 2$, we arrive at Eqs. (1819) where the noise terms $\xi_{n}$ are identified as $\xi_{n}(t)=e \zeta(t, t) / 2$. With the aid of Eq. (32) one can check that the noise correlator $\left\langle\xi_{n}\left(t_{1}\right) \xi_{n}\left(t_{2}\right)\right\rangle$ is given by the formula (9). This observation completes our derivation.

\section{SHOT NOISE}

The first and immediate application of our formalism concerns the analysis of the shot noise in arrays of scatterers and/or quantum dots in the absence of interactions. We will employ Eqs. 612 18 and evaluate the noise spectrum of quantum dot arrays in the zero frequency limit. Our procedure is similar to that applied in Ref. 13 to arrays of identical chaotic cavities.

From Eqs. (1213) we obtain

$$
f_{n}(E)=\left(1-a_{n}\right) f_{F}(E)+a_{n} f_{F}(E-e V),
$$

where we have defined $a_{n}=\sum_{j=1}^{n} R_{j} / R_{\Sigma}$. Substituting the result (39) into Eq. (6) we derive the noise spectrum for the $n$-th junction. In the zero frequency limit one finds

$$
\begin{array}{r}
\mathcal{S}_{n}=2\left\langle\left|\xi_{n}\right|_{\omega=0}^{2}\right\rangle=\left[1-a_{n}\left(1-a_{n}\right)-a_{n-1}\left(1-a_{n-1}\right)\right. \\
\left.-\beta_{n}\left(a_{n}-a_{n-1}\right)^{2}\right] \frac{4 T}{R_{n}}+\left[a_{n-1}\left(1-a_{n-1}\right)\right. \\
\left.+a_{n}\left(1-a_{n}\right)+\beta_{n}\left(a_{n}-a_{n-1}\right)^{2}\right] \frac{2 e V}{R_{n}} \operatorname{coth} \frac{e V}{2 T}
\end{array}
$$

Finally, we note that at sufficiently low frequencies the term with charges $q_{n}$ in the right-hand side of Eq. (19) can be neglected. In this limit the current fluctuations $\delta I$ in the whole array are related to the current and voltage fluctuations across the $n$-th scatterer as

$$
\delta I=\frac{\delta V_{n}}{R_{n}}+\xi_{n}
$$

where fluctuating voltages are subject to the constraint $\sum_{n=1}^{N} \delta V_{n}=0$. We obtain $\delta I=\left(1 / R_{\Sigma}\right) \sum_{n=1}^{N} R_{n} \xi_{n}$ and

$$
\mathcal{S}=\sum_{n=1}^{N} R_{n}^{2} \mathcal{S}_{n} / R_{\Sigma}^{2}
$$

Then with the aid of Eq. (40) we get

$$
\mathcal{S}=(1-\tilde{\beta}) \frac{4 T}{R_{\Sigma}}+\tilde{\beta} \frac{2 e V}{R_{\Sigma}} \operatorname{coth} \frac{e V}{2 T},
$$

where $\tilde{\beta}$ is the Fano factor for 1D arrays of chaotic quantum dots, which is obtained in the form

$$
\tilde{\beta}=\frac{1}{3}+\sum_{n=1}^{N} \frac{R_{n}^{3}}{R_{\Sigma}^{3}}\left(\beta_{n}-\frac{1}{3}\right) .
$$

For arrays of diffusive scatterers with $\beta_{n}=1 / 3$ one obviously gets $\tilde{\beta}=1 / 3$ for all values $R_{n}$. For homogeneous arrays with $R_{n}=R$ and $\beta_{n}=\beta$ Eq. (43) yields

$$
\tilde{\beta}=\frac{1}{3}+\frac{1}{N^{2}}\left(\beta-\frac{1}{3}\right) \text {. }
$$

This result demonstrates that in the limit $N \rightarrow \infty$ an array of arbitrary - not necessarily diffusive - scatterers should behave as a diffusive conductor with $\tilde{\beta} \rightarrow 1 / 3$. In the case of identical transmissions for all conducting channels Eq. (44) reduces to that derived in Ref. 13 .

\section{GENERAL EXPRESSION FOR THE CURRENT}

Let us now turn to the calculation of the currentvoltage characteristics in the presence of electron-electron interactions. According to Eq. (21), in order to accomplish this goal it is necessary to evaluate the average value for the noise terms $\xi_{n}$. This average would vanish identically, $\left\langle\xi_{n}\right\rangle=0$, should there be no dependence between the fluctuating voltage $V_{n}$ and noise $\xi_{n}$. However, since such a dependence in general exists, the averages $\left\langle\xi_{n}\right\rangle$ differ from zero and the interaction correction remains finite.

In this paper we will restrict ourselves to the perturbation theory and provide a general expression for the interaction correction to the $I-V$ curve. In what follows we will consider noise as a small perturbation, in which case in the leading approximation fluctuations of the phase on $n$-th junction and the noise $\xi_{m}$ are related to each other by means of a simple formula

$$
\delta \varphi_{n}(t)=-\frac{1}{e} \sum_{m=1}^{N} \int d \tau K_{n m}(t-\tau) \xi_{m}(\tau) .
$$

An explicit expression for the function $K_{n m}(\tau)$ will be specified later. Now we only point out that due to the causality requirement one has $K_{n m}(\tau<0)=0$. Making use of the above relation in the lowest non-vanishing order one can express the average value of $\xi_{n}$ in the form

$$
\begin{aligned}
& \left\langle\xi_{n}(t)\right\rangle=\sum_{m=1}^{N}\left\langle\int d t_{1} \frac{\delta \xi_{n}(t)}{\delta \varphi_{m}\left(t_{1}\right)} \delta \varphi_{m}\left(t_{1}\right)\right\rangle \\
& =-\frac{1}{e} \sum_{m, k=1}^{N} \int d t_{1} d t_{2} K_{m k}\left(t_{1}-t_{2}\right)\left\langle\frac{\delta \xi_{n}(t)}{\delta \varphi_{m}\left(t_{1}\right)} \xi_{k}\left(t_{2}\right)\right\rangle(.46)
\end{aligned}
$$


Here the derivative $\delta \xi_{n}(t) / \delta \varphi_{m}\left(t_{1}\right)$ accounts for the feedback of the phase fluctuations on the shot noise. Formally this effect is encoded in the Green function $G_{n}\left(t_{1}, t_{2}\right)$ which satisfies Eq. (15) and determines the noise correlator (9). In the lowest non-vanishing order in $\xi_{n}$ it is sufficient to employ Eq. (15) instead of (34). From the causality requirement one finds $\delta \xi_{n}(t) / \delta \varphi_{m}\left(t_{1}\right)=0$ for $t_{1}>t$. Utilizing this property together with a similar one for the function $K_{m k}(t)$ and making use of the fact that the noise variables for different scatterers are uncorrelated one can rewrite Eq. (46) in the form

$$
\left\langle\xi_{n}\right\rangle=-\frac{1}{e} \sum_{m=1}^{N} \int d t_{1} d t_{2} K_{m n}\left(t_{1}-t_{2}\right) \frac{\delta\left\langle\xi_{n}(t) \xi_{n}\left(t_{2}\right)\right\rangle}{\delta \varphi_{m}\left(t_{1}\right)}(47)
$$

In order to evaluate the functional derivative in Eq. (47) it is necessary to resolve Eq. (15). Proceeding perturbatively in $\delta \varphi_{m}$ and expressing the Green function $G_{n}\left(t_{1}, t_{2}\right)$ in the form

$$
G_{n}\left(t_{1}, t_{2}\right)=\mathrm{e}^{-i \Phi_{n}\left(t_{1}\right)+i \Phi_{n}\left(t_{2}\right)} U_{n}\left(t_{1}, t_{2}\right),
$$

we arrive at the equation for the function $U_{n}\left(t_{1}, t_{2}\right)$

$$
\begin{array}{r}
\frac{4 \pi}{\delta_{n}}\left(\frac{\partial}{\partial t_{1}}+\frac{\partial}{\partial t_{2}}\right) U_{n}\left(t_{1}, t_{2}\right)=-\left(g_{n}+g_{n+1}\right) U_{n}\left(t_{1}, t_{2}\right) \\
+g_{n} \mathrm{e}^{i \varphi_{n}\left(t_{1}\right)-i \varphi_{n}\left(t_{2}\right)} U_{n-1}\left(t_{1}, t_{2}\right) \\
+g_{n+1} \mathrm{e}^{-i \varphi_{n+1}\left(t_{1}\right)+i \varphi_{n+1}\left(t_{2}\right)} U_{n+1}\left(t_{1}, t_{2}\right)(49)
\end{array}
$$

With the aid of this equation we obtain

$$
\begin{gathered}
\delta U_{n}\left(t_{1}, t_{2}\right)=\frac{i \delta_{n}}{4 \pi} \mathrm{e}^{i \Phi_{n}\left(t_{1}\right)-i \Phi_{n}\left(t_{2}\right)} \sum_{m=1}^{N} \int d \tau g_{m} \delta \varphi_{m}(\tau) \\
\times\left\{\left(D_{n m}\left(t_{1}-\tau\right)-D_{n m}\left(t_{2}-\tau\right)\right) G_{m-1}^{(0)}\left(t_{1}-t_{2}\right)\right. \\
\left.-\left(D_{n, m-1}\left(t_{1}-\tau\right)-D_{n, m-1}\left(t_{2}-\tau\right)\right) G_{m}^{(0)}\left(t_{1}-t_{2}\right)\right\}(50)
\end{gathered}
$$

Here $G_{n}^{(0)}\left(t_{1}-t_{2}\right)$ is the solution of Eq. (15) obtained for $\delta \dot{\Phi}_{n}=0$. It reads

$$
G_{n}^{(0)}(s)=\frac{-i T}{\sinh \pi T s}\left(1-a_{n}+a_{n} \mathrm{e}^{-i e V s}\right),
$$

where the coefficients $a_{n}$ are defined after Eq. (39) and the "diffuson" $D_{n m}(t)$ satisfies the equation

$$
\begin{aligned}
\frac{\partial D_{n, m}}{\partial t}= & \frac{\delta_{n}}{4 \pi}\left(g_{n} D_{n-1, m}+g_{n+1} D_{n+1, m}\right. \\
& \left.-\left(g_{n}+g_{n+1}\right) D_{n, m}\right)+\delta_{n m} \delta(t)
\end{aligned}
$$

with the boundary conditions

$$
D_{0, m}=D_{N, m}=D_{n, 0}=D_{n, N}=0 .
$$

As before, due to causality one has $D_{n m}(t)=0$ for $t<0$.

With the aid of the above expressions we get

$$
\begin{aligned}
& \frac{\delta G_{n}\left(t, t_{2}\right)}{\delta \varphi_{m}\left(t_{1}\right)}=-i G_{n}^{(0)}\left(t-t_{2}\right) \\
& \times\left[\delta\left(t-t_{1}\right)-\delta\left(t_{2}-t_{1}\right)\right] \theta(n-m+0) \\
& +\frac{i \delta_{n} g_{m}}{4 \pi}\left\{\left[D_{n m}\left(t-t_{1}\right)-D_{n m}\left(t_{2}-t_{1}\right)\right] G_{m-1}^{(0)}\left(t-t_{2}\right)\right. \\
& \left.\quad-\left[D_{n, m-1}\left(t-t_{1}\right)-D_{n, m-1}\left(t_{2}-t_{1}\right)\right] G_{m}^{(0)}\left(t-t_{2}\right)\right\}(54)
\end{aligned}
$$

This equation in combination with Eqs. (947) enables one to evaluate the derivative $\delta\left\langle\xi_{n}(t) \xi_{n}\left(t_{2}\right)\right\rangle / \delta \varphi_{m}\left(t_{1}\right)$ and derive the final expression for the current. With the aid of Eq. (21) we obtain

$$
I=\frac{V}{R_{\Sigma}}+\delta I
$$

where $\delta I$ is the interaction correction which can be split into two parts $\delta I=\delta I_{1}+\delta I_{2}$ :

$$
\begin{aligned}
& \delta I_{1}= \frac{1}{4 \pi^{2} e R_{\Sigma}} \sum_{n, m=1}^{N} g_{m} \int d t d t^{\prime} \frac{\pi^{2} T^{2} \sin e V t}{\sinh ^{2} \pi T t} \\
& \times K_{m n}\left(t-t^{\prime}\right)\left[\delta_{n-1}\left(a_{m-1}-a_{n-1}\right) D_{n-1, m}\left(t^{\prime}\right)\right. \\
&-\delta_{n-1}\left(a_{m}-a_{n-1}\right) D_{n-1, m-1}\left(t^{\prime}\right) \\
&+\delta_{n}\left(a_{m-1}-a_{n}\right) D_{n, m}\left(t^{\prime}\right) \\
&\left.-\delta_{n}\left(a_{m}-a_{n}\right) D_{n, m-1}\left(t^{\prime}\right)\right] \\
& \delta I_{2}=-\frac{1}{\pi e R_{\Sigma}^{2}} \sum_{n, m=1}^{N} \beta_{n} R_{n} \int d t d t^{\prime} \frac{\pi^{2} T^{2} \sin e V t}{\sinh ^{2} \pi T t} \\
& \times K_{m n}(\left.t-t^{\prime}\right)\left[\delta_{n m} \delta\left(t^{\prime}\right)-\frac{\delta_{n-1} g_{m}}{4 \pi}\left(D_{n-1, m-1}\left(t^{\prime}\right)\right.\right. \\
&\left.\left.-D_{n-1, m}\left(t^{\prime}\right)\right)-\frac{\delta_{n} g_{m}}{4 \pi}\left(D_{n m}\left(t^{\prime}\right)-D_{n, m-1}\left(t^{\prime}\right)\right)\right]
\end{aligned}
$$

Eqs. (55.57) represent our general result for the $I-V$ curve of a $1 d$ array of metallic quantum dots in the presence of interactions. One can verify that in the particular case of two scatterers or, equivalently, for a single chaotic quantum dot, Eqs. (55 57) reduce to the expressions derived in Ref. 8 by means of a different approach. Let us also note that the expression for the function $K_{m n}(t)$ is determined by the solution of Eqs. (17 19) under the constraint (20). Below we will explicitly find this solution for the specific case of homogeneous arrays of quantum dots.

\section{HOMOGENEOUS 1D ARRAY}

Consider an array formed by the scatterers and quantum dots with identical parameters. In what follows we set $C_{n}=C, C_{g n}=C_{g}, g_{n}=g, \beta_{n}=\beta, R_{n}=R$, $\delta_{n}=\delta$. In this case it is straightforward to derive the exact expressions for the functions $K_{m n}(t)$ and $D_{n m}$. These expressions read

$$
\begin{aligned}
& K_{m n}(t)=\frac{2 e^{2}}{N} \sum_{q=1}^{N-1} \int \frac{d \omega}{2 \pi} \frac{\mathrm{e}^{-i \omega t}}{-i \omega+0} Z_{\omega q} \\
& \times \cos \left(\frac{\pi q n}{N}-\frac{\pi q}{2 N}\right) \cos \left(\frac{\pi q m}{N}-\frac{\pi q}{2 N}\right),
\end{aligned}
$$

where we defined the impedance $Z_{\omega q}$

$$
Z_{\omega q}=\frac{1}{\left(-i \omega+\frac{1-\cos \frac{\pi q}{N}}{\tau_{D}}\right)\left(C+\frac{C_{g}}{2\left(1-\cos \frac{\pi q}{N}\right)}\right)+\frac{1}{R}},
$$


and

$$
\begin{aligned}
D_{n m} & =\frac{2}{N} \sum_{q=1}^{N-1} \int \frac{d \omega}{2 \pi} \mathrm{e}^{-i \omega t} D_{\omega q} \sin \frac{\pi q n}{N} \sin \frac{\pi q m}{N} \\
D_{\omega q} & =\frac{1}{-i \omega+\frac{1-\cos (\pi q / N)}{\tau_{D}}}
\end{aligned}
$$

Here and below $\tau_{D}=2 \pi / g \delta$ stands for the electron dwell time in a single quantum dot and the coefficients $a_{n}$ reduce to $a_{n}=n / N$. Making use of the property $K_{m n}(t)=K_{n m}(t)$ and $D_{m n}(t)=D_{n m}(t)$ we obtain

$$
\begin{aligned}
\delta I_{1}= & -e \sum_{q=1}^{N-1} \frac{1+\cos \frac{\pi q}{N}}{2 \tau_{D} R N^{2}} \int \frac{d \omega}{2 \pi} \operatorname{Im}\left(Z_{\omega q} D_{\omega q}^{2}\right) B(\omega, V, T) \\
& +e \sum_{q=1}^{N-1} \frac{1+\cos \frac{\pi q}{N}}{\tau_{D}^{2} R N^{3}} \operatorname{Im} \int \frac{d \omega}{2 \pi} Z_{\omega q} D_{\omega q}^{3} B(\omega, V, T) \\
& \times \frac{1-(-1)^{q} u^{N}(\omega)}{1+(-1)^{q} u^{N}(\omega)} \sqrt{-i \omega \tau_{D}\left(2-i \omega \tau_{D}\right)}
\end{aligned}
$$

and

$$
\delta I_{2}=-\frac{e \beta}{2 N^{2} R} \sum_{q=1}^{N-1} \int \frac{d \omega}{2 \pi} \operatorname{Im}\left(Z_{\omega q} D_{\omega q}\right) B(\omega, V, T),
$$

where $B(\omega, V, T)=\sum_{ \pm}(e V \pm \omega) \operatorname{coth} \frac{\omega \pm e V}{2 T}$ and $u(\omega)=$ $1-i \omega-\sqrt{\left(1-i \omega \tau_{D}\right)^{2}-1}$. We observe that the second contribution to the interaction correction $\delta I_{2}$ scales with the Fano factor $\beta$ of individual scatterers and, hence, vanishes for $\beta \rightarrow 0$. At the same time the first contribution $\delta I_{1}$ does not depend on $\beta$, i.e. it is universal for any type of scatterers. We also note that the term $\delta I_{1}$ differs from zero for all $N>2$ but vanishes identically in the case of two scatterers $N=2$.

The frequency integrals in Eqs. (61) and (62) can be performed exactly with the result

$$
\begin{aligned}
\delta I= & -\frac{2 T e}{N^{2}} \sum_{q=1}^{N-1}\left[\beta+\frac{\cos \frac{\pi q}{N}}{4 \sin ^{2} \frac{\pi q}{2 N}}\right]\left[W\left(\frac{2 \sin ^{2} \frac{\pi q}{2 N}}{\pi T R\left(4 C \sin ^{2} \frac{\pi q}{2 N}+C_{g}\right)}+\frac{\sin ^{2} \frac{\pi q}{2 N}}{\pi T \tau_{D}}+\frac{i e V}{2 \pi T}\right)-W\left(\frac{\sin ^{2} \frac{\pi q}{2 N}}{\pi T \tau_{D}}+\frac{i e V}{2 \pi T}\right)\right] \\
& -\frac{4 T e}{N^{4}} \sum_{p, q=1}^{N-1} \frac{\left(1-(-1)^{p+q}\right) \sin ^{2} \frac{\pi q}{N} \sin ^{2} \frac{\pi p}{N}}{\left(\cos \frac{\pi q}{N}-\cos \frac{\pi p}{N}\right)^{3}\left(2 \sin ^{2} \frac{\pi p}{2 N}+\frac{R}{2 \tau_{D}}\left(4 C \sin ^{2} \frac{\pi p}{2 N}+C_{g}\right)\left(\cos \frac{\pi q}{N}-\cos \frac{\pi p}{N}\right)\right)} \\
& \times\left[W\left(\frac{2 \sin ^{2} \frac{\pi p}{2 N}}{\pi T R\left(4 C \sin ^{2} \frac{\pi p}{2 N}+C_{g}\right)}+\frac{\sin ^{2} \frac{\pi p}{2 N}}{\pi T \tau_{D}}+\frac{i e V}{2 \pi T}\right)-W\left(\frac{\sin ^{2} \frac{\pi q}{2 N}}{\pi T \tau_{D}}+\frac{i e V}{2 \pi T}\right)\right] .
\end{aligned}
$$

Here we defined the function $W(x)=\operatorname{Im}[x \Psi(1+x)]$, where $\Psi(x)$ is the digamma function. Eq. 63) is the exact expression for the leading (in $1 / g$ ) interaction correction to the current valid both in linear and non-linear in voltage regimes and for any number of scatterers $N$ in the system.

Let us consider a physically important limit of relatively large metallic quantum dots with $R C, R C_{g} \ll \tau_{D}$. Making use of this inequality one can significantly simplify the general result (63) and find

$$
\begin{aligned}
& \delta I=-\frac{2 T e}{N^{2}} \sum_{q=1}^{N-1}\left[\beta W\left(\frac{2 \sin ^{2} \frac{\pi q}{2 N}}{\pi T R\left(4 C \sin ^{2} \frac{\pi q}{2 N}+C_{g}\right)}+\frac{i e V}{2 \pi T}\right)\right. \\
& \left.-\left[\beta-\frac{1-\frac{2\left(1-(-1)^{q}\right)}{N^{2}} \cot ^{2} \frac{\pi q}{2 N}}{1-\cos \frac{\pi q}{N}}\right] W\left(\frac{\sin ^{2} \frac{\pi q}{2 N}}{\pi T \tau_{D}}+\frac{i e V}{2 \pi T}\right)\right] .
\end{aligned}
$$

In the linear in voltage regime the above expression yields the result for the zero bias conductance of the array $G=$
$1 / N R+\delta G$. The interaction correction $\delta G$ takes the form

$$
\begin{aligned}
\delta G & =-\frac{e^{2}}{\pi N^{2}} \sum_{q=1}^{N-1}\left[\beta L\left(\frac{2 \sin ^{2} \frac{\pi q}{2 N}}{\pi T R\left(4 C \sin ^{2} \frac{\pi q}{2 N}+C_{g}\right)}\right)\right. \\
& \left.-\left(\beta-\frac{1-\frac{2\left(1-(-1)^{q}\right)}{N^{2}} \cot ^{2} \frac{\pi q}{2 N}}{1-\cos \frac{\pi q}{N}}\right) L\left(\frac{\sin ^{2} \frac{\pi q}{2 N}}{\pi T \tau_{D}}\right)\right],
\end{aligned}
$$

where we have defined $L(x)=\Psi(1+x)+x \Psi^{\prime}(1+x)$.

Let us now briefly analyze the above results in various limits. The case $N=2$ was already considered in details in Ref. [8, here we will concentrate on the behavior of quantum dot arrays containing many scatterers $N \gg 1$. In this case the expression for the interaction correction (65) can be further simplified. In the high temperature limit $T R C_{m} \gg 1$ (where $C_{m}=\min \left[C, C_{g}\right]$ ) we obtain

$$
\delta G=-\frac{e^{2} \beta}{6 N T R C}\left(1-\sqrt{\frac{C_{g}}{C_{g}+4 C}}\right) .
$$

At intermediate temperatures $1 / R C, 1 / R C_{g} \gg T \gg$ 


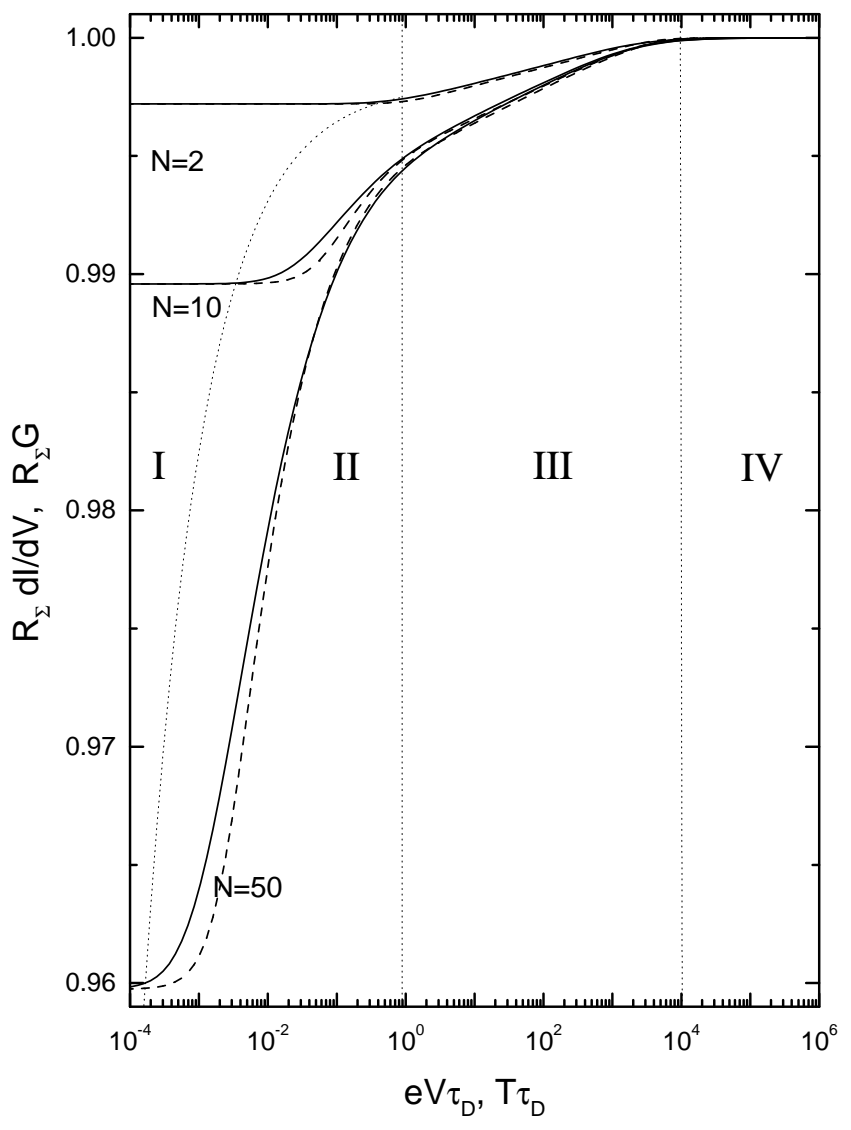

FIG. 2: The linear conductance $G$ (solid lines) as a function of temperature $T$ together with the differential conductance $d I / d V$ (dashed lines) as a function of the applied voltage $V$ at $T=0$. The results are obtained from Eq. (63) for $g=1000$, $\beta=1 / 3, \tau_{D} / R C=10^{4}, C_{g} / C=2.5$ and for three different numbers of barriers: $N=2,10$ and 50 . We identify four different regimes (the boundaries between them are shown by dotted lines): (I) saturation regime $e V, T \lesssim \pi^{2} / 2 N^{2} \tau_{D}$, Eq. [69), (II) diffusive regime $\pi^{2} / 2 N^{2} \tau_{D} \lesssim e V, T \lesssim 1 / \tau_{D}$, Eqs. 6870), (III) logarithmic regime of almost independent barriers $1 / \tau_{D} \lesssim e V, T \lesssim 1 / R C$, Eqs. (6771) and (IV) high temperature (classical) regime $e V, T \gtrsim 1 / R C$, Eq. (66).

$1 / \tau_{D}$ Eq. 65) yields

$$
\delta G \approx-\frac{e^{2} \beta}{\pi N}\left[\ln \frac{1}{2 \pi T R C^{*}}+1\right]
$$

where $C^{*}=\left(\sqrt{C_{g}}+\sqrt{C_{g}+4 C}\right)^{2} / 4$. In the interval $\pi^{2} / 2 N^{2} \tau_{D} \ll T \ll 1 / \tau_{D}$ we find

$$
\begin{aligned}
& \delta G \approx-\frac{e^{2} \beta}{\pi N} \ln \frac{2 \tau_{D}}{R C^{*}}-\frac{e^{2}}{\pi N} \frac{3 \zeta(3 / 2)}{4 \sqrt{\pi T \tau_{D}}} \\
& +\frac{e^{2}}{\pi N}\left[1+\left(\beta-\frac{1}{4}\right)\left(1-\frac{\gamma}{2}\right) \sqrt{\pi T \tau_{D}}+\frac{\pi}{3 N T \tau_{D}}\right]
\end{aligned}
$$

where $\gamma \simeq 0.577$ is the Euler constant. Finally, in the limit of very low temperatures, $T \ll \pi^{2} / 2 N^{2} \tau_{D}$, the cor- rection to the conductance saturates, and we get

$$
\delta G \approx-\frac{e^{2} \beta}{\pi N} \ln \frac{2 \tau_{D}}{R C^{*}}-\left(0.368-\frac{1}{N}\right) \frac{e^{2}}{\pi} .
$$

Consider now the nonlinear regime $e|V| \gg T$ in which case the $I-V$ curve is determined by Eq. (63) (or (64)). We will again consider the limit $N \gg 1$ and make use of Eq. (64). At very small voltages and temperatures, $e|V| \ll \pi^{2} / 2 N^{2} \tau_{D}$, one finds $d I / d V=1 / N R+\delta G$, where $\delta G$ is again defined by Eq. (69). At higher voltages, $\pi^{2} / 2 N^{2} \tau_{D} \ll e|V| \ll 1 / \tau_{D}$ we obtain

$$
\frac{d I}{d V}=\frac{1}{N R}-\frac{e^{2} \beta}{\pi N} \ln \frac{2 \tau_{D}}{R C^{*}}-\frac{e^{2}}{\pi N} \frac{1}{\sqrt{2 e|V| \tau_{D}}} .
$$

At even higher voltage, $1 / \tau_{D} \ll e|V| \ll 1 / R C^{*}$, the differential conductance takes the form

$$
\frac{d I}{d V}=\frac{1}{N R}-\frac{e^{2} \beta}{\pi N} \ln \frac{1}{e|V| R C^{*}} .
$$

The linear conductance $G$ as a function of temperature and the differential conductance $d I / d V$ at $T \rightarrow 0$ as a function of the applied voltage are depicted in Fig. 2 for different number of scatterers $N$ in the system. One observes that both quantities (as functions of corresponding variables) demonstrate a very similar behavior. In both cases four different regimes can be distinguished, as it was already specified above. Further discussion of these results is postponed to Sec. VIII.

\section{EFFECT OF ENVIRONMENT}

In order to complete our analysis let us also include the effect of external leads into consideration. For simplicity we will follow the standard procedure and assume the leads impedance to be purely Ohmic. This procedure amounts to replacing Eq. (20) by a more general one,

$$
\sum_{j=1}^{N} V_{j}+I R_{S}=V_{x}
$$

where $V_{x}$ is the voltage applied to the whole system "array+leads", $R_{S}$ is the resistance of the leads and $I=C \dot{V}_{n}+I_{n}$ is the current flowing through the leads. Due to current conservation the scatterer number $n$ can be chosen arbitrarily here. Eq. (172) is solved together with Eqs. 914171821 in exactly the same way as it was done above in the absence of the shunt. As a result, the effect of $R_{S}$ is accounted for by means of a simple replacement

$$
K_{n m}(t) \longrightarrow K_{n m}(t)+K_{S}(t),
$$

where $K_{n m}(t)$ is defined in Eq. (58) and

$$
K_{S}(t)=\frac{e^{2}}{N} \int \frac{d \omega}{2 \pi} \frac{\mathrm{e}^{-i \omega t}}{(-i \omega+0)\left(-i \omega+\frac{1}{R}+\frac{N}{R_{S}}\right)}
$$


Note that the function $K_{S}(t)$ does not depend on $n$ and $m$. After such a replacement the new expression for $K_{n m}(t)$ should be substituted into Eqs. (56157) and we arrive at the result

$$
I=\frac{V}{R_{\Sigma}}+\delta I+\delta I_{S},
$$

where $V=\sum_{j=1}^{N}\left\langle V_{j}\right\rangle$ is the average voltage across the array and $\delta I$ is defined in Eq. (63). The general expression for the additional term $\delta I_{S}$ is rather cumbersome and will not be presented here. Below we will only address the effect of external leads on the linear conductance in the low temperature limit $T<\pi^{2} / 2 N^{2} \tau_{D}$. It turns out that for non-zero $R_{S}$ - similarly to the case of single quantum $\operatorname{dots}^{8}$ - the conductance saturation is lifted and the result (69) becomes incomplete. Taking into account the shunt contribution to the current $\delta I_{S}$, one finds

$$
\begin{aligned}
G \approx & \frac{1}{N R}-\frac{e^{2} \beta}{\pi N} \ln \frac{2 \tau_{D}}{R C^{*}}-\left(0.368-\frac{1}{N}\right) \frac{e^{2}}{\pi} \\
& -\frac{e^{2} \tilde{\beta}}{\pi} \frac{R_{S}}{R_{S}+N R} \ln \frac{\pi^{2}}{2 N^{2} T \tau_{D}},
\end{aligned}
$$

where

$$
\tilde{\beta}=\frac{\beta}{N^{2}}+\frac{1}{N^{4}} \sum_{q=1}^{N-1} \frac{\left(1-(-1)^{q}\right) \cos ^{2} \frac{\pi q}{2 N}}{\sin ^{4} \frac{\pi q}{2 N}} .
$$

The sum in (77) is evaluated exactly and just yields the Fano factor of the array (44). Thus, in the presence of an external shunt the conductance keeps decreasing logarithmically with $T$ even at very low temperatures. As before, this logarithmic contribution scales linearly with the total Fano factor of the array $\tilde{\beta}$ which tends to the universal value $1 / 3$ in the limit of large $N$. This result is in the agreement with our previous findings 1.8 and once again emphasizes a direct relation between shot noise and interaction effects in disordered conductors.

\section{DISCUSSION}

In this paper we have proposed a general model which embraces virtually any type of disordered conductors and allows to account for Coulomb interaction effects in electron transport through such conductors. Exploiting an intimate relation between shot noise and interaction effects, in Sec. II and III we derived a closed set of Langevin-type of equations which allow to conveniently study electron transport in the presence of electronelectron interactions ${ }^{18}$. The key idea of our approach is to account for modifications of the shot noise due to nonequilibrium effects and to self-consistently describe these effects and their impact on fluctuating charges and voltages inside the conductor. For the sake of definiteness here we focused our attention on quasi-1D conductors, however one can trivially extend the whole analysis to
$2 \mathrm{D}$ and $3 \mathrm{D}$ conductors as well. This generalization will be carried out elsewhere.

The technique developed in this paper allows to obtain a general formula for the interaction correction to the current, Eq. 63), which contains all the results derived previously for various quasi-0D and quasi-1D disordered conductors and extends these results to yet unexplored regimes. At sufficiently high energies (exceeding the inverse dwell time of a single quantum dot $1 / \tau_{D}$ ) the scatterers behave as effectively independent ones, and one can identify two different regimes (regimes III and IV in Fig. 2) described by Eqs. (677166). At such energies the interaction correction scales with the Fano factor $\beta$ of individual scatterers and in a wide interval of energies depends logarithmically on temperature or voltage. For a special case of tunnel barriers $\beta \rightarrow 1$ our results reduce to those derived in Refs. 714 , while in the limit of ballistic contacts with $\beta \rightarrow 0$ (or, equivalently, diffusive wires with point-like impurities) the interaction correction turns out to be negligibly small in this regime.

At energies below $1 / \tau_{D}$ (regime II) scatterers located sufficiently close to each other become effectively correlated. The number of such scatterers $N_{\text {eff }}$ in one "correlated" segment of the array grows with decreasing temperature (or voltage) as $N_{\text {eff }} \sim 1 / \sqrt{T \tau_{D}}$ (or $\left.N_{\text {eff }} \sim 1 / \sqrt{e V \tau_{D}}\right)$. In this regime the system can be viewed as a chain of $\sim N / N_{\text {eff }}$ segments, each of them now playing the role of a "new" independent scatterer with an effective conductance $g_{\text {eff }} \sim g / N_{\text {eff }} \sim g \sqrt{T \tau_{D}}$ (or $\left.\sim g \sqrt{e V \tau_{D}}\right)$. Then the results 7,14 can be applied again, in the corresponding expression for the interaction correction one should only substitute $g_{\text {eff }}$ instead of $g$. In this case the logarithmic dependence of the interaction correction on temperature/voltage drops out ${ }^{19}$ and, e.g., for the linear conductance one finds $\delta G / G \sim-\beta_{\text {eff }} / g_{\text {eff }}$, where $\beta_{\text {eff }}$ is the Fano factor of a segment with $N_{\text {eff }}$ scatterers. According to Eq. (44), for sufficiently large $N_{\text {eff }} \gg 1$ the factor $\beta_{\text {eff }}$ approaches the universal value $1 / 3$, and we obtain $\delta G / G \sim-1 / g \sqrt{T \tau_{D}}$ in agreement with the well known result ${ }^{11}$ and also with our rigorous formula (68) which - in addition - contains a temperature-independent contribution $\propto \beta$ coming from high energy modes. Finally, as $N_{\text {eff }}$ approaches $N$ the system conductance either saturates (for $R_{S} \rightarrow 0$, regime I) or crosses over to the low energy logarithmic regime (76) caused by additional voltage fluctuations across the array due to non-zero external shunt resistance $R_{S}$.

It is also straightforward to establish a direct relation between the results derived here and those obtained diagrammatically in the linear in voltage regime ${ }^{10,11}$. By setting $N \rightarrow \infty$ and $\beta \rightarrow 1$ from Eq. (65) we reproduce the results 10 for the interaction correction in tunnel junction arrays, while in the limit $\beta \rightarrow 0$ the latter equation yields the standard result ${ }^{11}$ for diffusive wires 20 . The same equivalence can be observed at the level of general expressions (6162) considered in the limit $e V \ll T$, $N \rightarrow \infty$ and for $\tau_{D} \gg R C, R C_{g}$. For $\beta=1$ the result 10 follows from the sum of two terms (61) and (62), while 
for $\beta=0$ the second contribution (62) vanishes identically and the result ${ }^{11}$ is obtained only from the first term (61). These observations demonstrate that the reduction of our model to one for diffusive wires with point-like impurities is achieved by setting $\beta \rightarrow 0$. In the latter case $N$ coincides with the total number of impurities in the wire.

At last, let us briefly summarize the applicability conditions for our results. As it was already discussed above, our Langevin approach is justified in the metallic limit $g_{n} \gg 1$. Under this condition our technique should account for all essential processes except for subtle instanton effects which may show up only at exponentially low energies. An obvious necessary (though possibly not sufficient) validity condition of our results derived in the linear in voltage regime is $\delta G / G \ll 1$. While at high enough temperatures this inequality is automatically fulfilled in the metallic limit $g_{n} \gg 1$, at the lowest energies/temperatures a much more stringent condition $g_{\Sigma}=2 \pi / e^{2} R_{\Sigma} \gg 1$ has to be satisfied. The latter condition is inevitably violated for large number of scatterers $N$ in which case a non-perturbative analysis becomes necessary in the low energy limit. This analysis is beyond the frames of the present paper. In the non-linear regime and at sufficiently high voltages the applicability range of our results can be additionally restricted by electron heating effects which we do not address in this work.

\section{Acknowledgements}

We are grateful to D.A. Bagrets and S.V. Sharov for stimulating discussions. This work is part of the Kompetenznetz "Funktionelle Nanostructuren" supported by the Landestiftung Baden-Württemberg gGmbH and of the STReP "Ultra-1D" supported by the EU.
1 D.S. Golubev and A.D. Zaikin, Phys. Rev. Lett. 86, 4887 (2001).

2 A.V. Galaktionov, D.S. Golubev and A.D. Zaikin, Phys. Rev. B 68, 085317 (2003); ibid., 68, 235333 (2003).

3 M. Kindermann and Yu.V. Nazarov, Phys. Rev. Lett. 91, 136802 (2003).

4 D.A. Bagrets and Yu.V. Nazarov, cond-mat/0304339

${ }^{5}$ L.S. Levitov, H. Lee, and G.B. Lesovik, J. Math. Phys. 37, 4845 (1996).

6 Ya.M. Blanter and M. Büttiker, Phys. Rep. 336, 1 (2000).

7 D.S. Golubev and A.D. Zaikin, Phys. Lett. A169, 475 (1992); Sh. Farhangfar, R.S. Poikolainen, J.P. Pekola, D.S. Golubev, and A.D. Zaikin, Phys. Rev. B 63, 075309 (2001).

8 D.S. Golubev and A.D. Zaikin, Phys. Rev. B 69, 0353XX (2004); cond-mat/0307161

9 P. Schwab and R. Raimondi, Eur. Phys. J. B 30, 5 (2002).

10 I.S. Beloborodov, K.B. Efetov, A.V. Lopatin, and V.M. Vinokur, Phys. Rev. Lett. 91, 246801 (2003).

11 B.L. Altshuler and A.G. Aronov, in Electron-Electron Interaction in Disordered Systems, ed. by A.L. Efros and M. Pollak (North-Holland, Amsterdam, 1985).

12 M.J.M. de Jong and C.W.J. Beenakker, Phys. Rev. B 51, 16867 (1995).

13 S. Oberholzer, E.V. Sukhorukov, C. Strunk, and C.
Schönenberger, Phys. Rev. B 66, 233304 (2002).

14 G. Göppert and H. Grabert, Eur. Phys. J. B 16, 687 (2000).

15 A. Kamenev and A. Andreev, Phys. Rev. B 60, 2218 (1999).

16 G. Schön and A.D. Zaikin, Phys. Rep. 198, 237 (1990).

17 D.S. Golubev and A.D. Zaikin, Phys. Rev. B 59, 9195 (1999).

18 In order to avoid misunderstandings we would like to emphasize again that our present approach accounts for the interaction correction to the $I-V$ curve and is not meant to describe weak localization effects and interaction-induced dephasing ${ }^{11,17}$ which we do not address in this work.

19 In this case the combination $T \tau_{D}^{\text {eff }}$ occurs under the logarithm, where $\tau_{D}^{\text {eff }}$ is the total dwell time for $N_{\text {eff }}$ scatterers. This combination reduces to a temperature independent constant of order unity since $\tau_{D}^{\text {eff }} \sim 1 / T$.

${ }^{20}$ In order to observe the equivalence between our Eq. 65 in the limit $\beta \rightarrow 0$ and the results ${ }^{11}$ for the interaction correction in quasi-1D diffusive wires it suffices to introduce the diffusion coefficient $D=a^{2} / 2 \tau_{D}$, where $a$ is the size of a single quantum dot. 